\title{
Extrarenal malignancy and the nephrotic syndrome
}

\author{
J. M. HEATON, M. A. MENZIN, AND D. N. CARNEY \\ From the Department of Pathology, Trinity College, and the Meath Hospital, Dublin
}

SYNOPSIS The clinical and histological findings are described of a patient who presented with the $\stackrel{\circ}{-}$ nephrotic syndrome and was found at necropsy to have a bronchial carcinoma. Amyloidosis, renal $\vec{\omega}$ vein thrombosis, and neoplastic infiltration of the kidneys were excluded. The kidneys showed a $\frac{\Omega}{\circ}$ diffuse glomerulonephritis of mesangiocapillary type. The possible aetiological association between these two conditions is discussed.

The association of the nephrotic syndrome with extrarenal malignancy is not an uncommon one (Lee et al, 1966; Lewis et al, 1971; da Costa et al, 1974). The vast majority of renal disease in relation to malignancy is that of membranous nephropathy.

Lee et al (1966) included one case of lobular glomerulonephritis in their series while Lewis et al (1971) reported one case of mesangiocapillary glomerulonephritis (MCGN) associated with carcinoma of the breast. MCGN has been reported on two occasions in lymphoma (Muggia and Ultmann, 1971; Hyman et al, 1973).

This appears to be the first report of MCGN associated with a bronchial neoplasm.

\section{Case Report}

A 50-year-old man was admitted to hospital in January 1974 with a history of chronic bronchitis. A diagnosis of nephrotic syndrome of uncertain aetiology was made together with mild right-sided heart failure secondary to extensive pulmonary fibrosis. Three months later he was readmitted with a blood urea of $200 \mathrm{mg} / 100 \mathrm{ml}$ and was dialysed. In view of his past history of pulmonary tuberculosis antituberculosis therapy was begun. His condition improved and he remained static for four months, when he developed superior vena caval obstruction. A chest $x$-ray at this time showed a mass in the right upper mediastinum. He died shortly afterwards.

\section{NECROPSY}

There was a necrotic tumour $(3 \times 3 \times 2 \mathrm{~cm})$ at the hilum of the right lung with abscess formation in the middle and lower lobes. The liver and regional nodes showed metastatic tumour deposits. The

Received for publication 24 June 1975. kidneys were grossly unremarkable. The renal veins $\stackrel{+}{\circ}$ were patent.

\section{Histology}

Sections from the lung showed a moderately well- $\frac{\AA}{3}$ differentiated squamous-cell carcinoma. The lung abscess was a pyogenic lesion; stains for acid-fast bacilli and fungi were negative.

The kidneys showed a diffuse glomerulonephrio of mesangiocapillary type with a lobular pattern 音 the glomerular tufts (fig 1). Electron microscopy of the renal tissue showed electron dense deposits in the mesangium and along the capillary basement membrane in a subendothelial position (fig 2). Mesangial $\stackrel{\circ}{\varnothing}$ interposition was also demonstrated. Immunofluorescent studies were not done.

\section{Discussion}

Loughridge and Lewis (1971) reported immuneo complex glomerulonephritis in a patient with the nephrotic syndrome and squamous carcinoma of the bronchus. At necropsy immunoglobulins eluted from the glomeruli reacted specifically with the patient's tumour cells. This study provided direct? evidence that the mechanism of renal disease in $>$ patients with cancer might be mediated by immune complexes composed of antitumour antibody and tumour antigen.

However, the incidence of the nephrotic syndromes does not appear to be higher in patients with known N tumour-associated antigens, for example, malignanto melanoma. Costanza et al (1974) have recentlyo demonstrated carcinoembryonic antigen-antibody complexes in glomeruli of a patient with colonic ${ }^{+}$ carcinoma and the nephrotic syndrome.

Following animal studies Germuth and Rodrigue 2 ? (1973) postulated that the anatomical localization of 


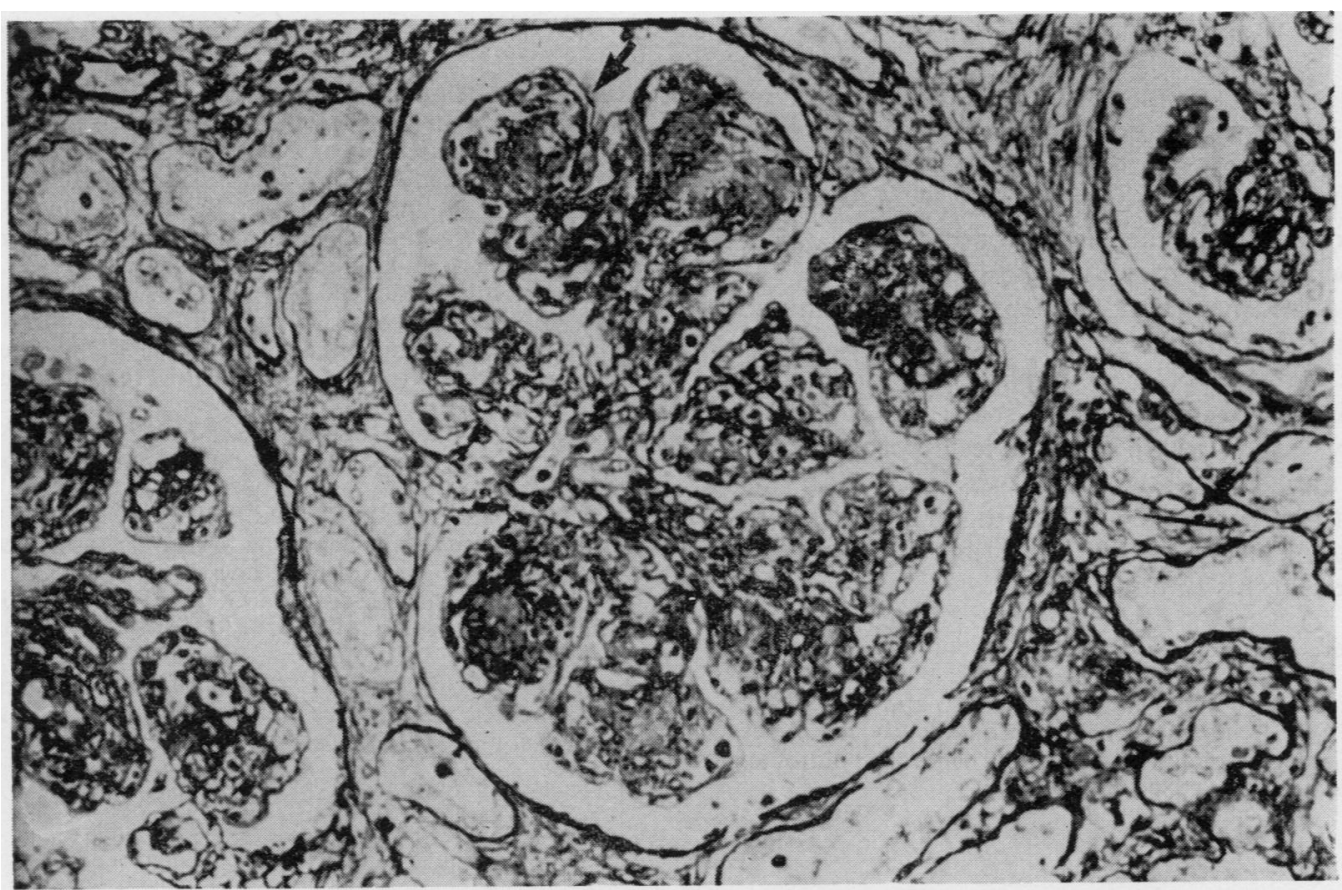

Fig 1 Mesangiocapillary glomerulonephritis with a 'double contour' (arrow) appearance of the glomerular capillary walls due to mesangial interposition. PASM $\times 300$

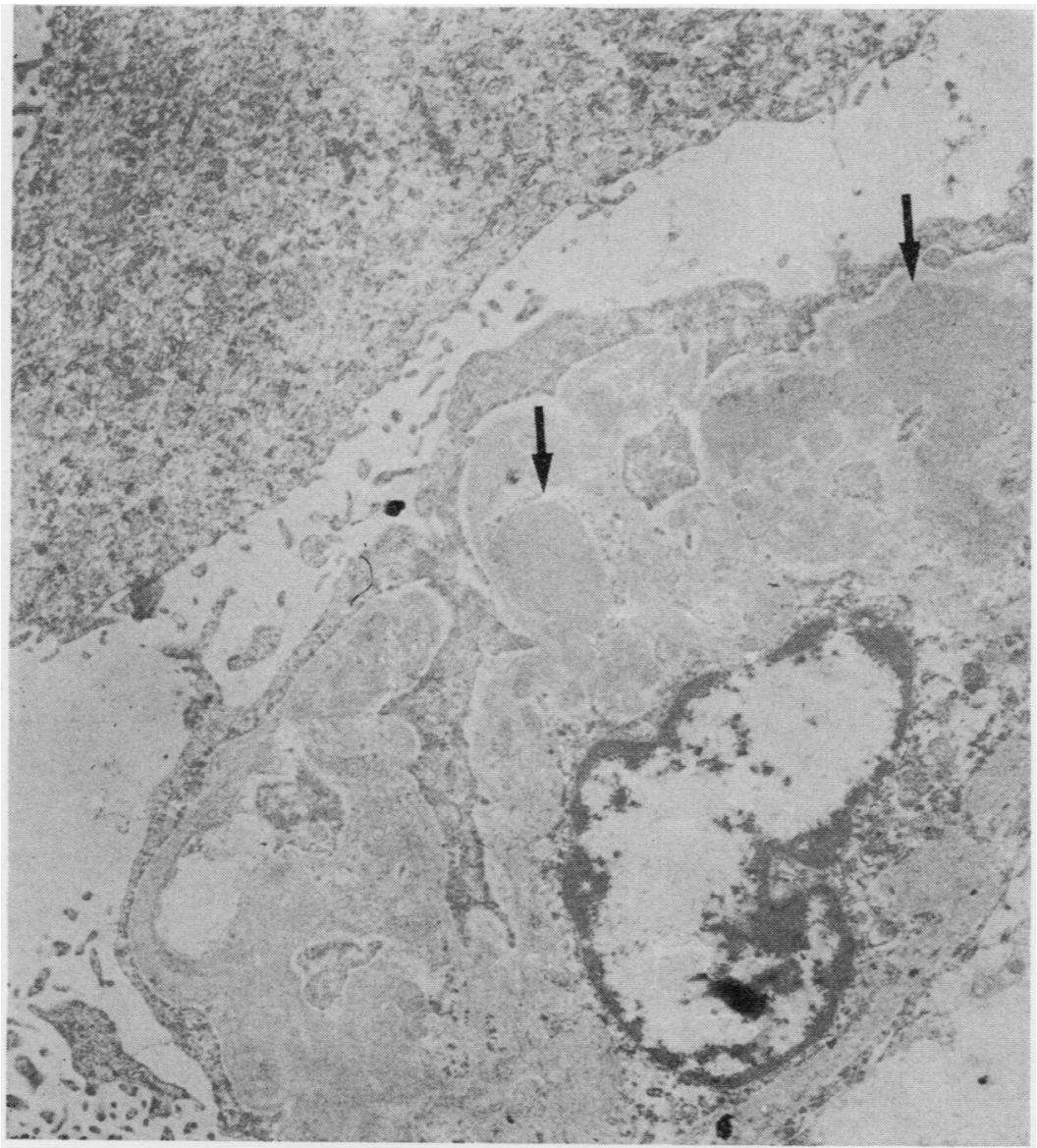

Fig 2 Electron micrograph showing glomerular capillary loop with subendo thelial electron dense deposits (arrows). $\times 12,000$ 
immune complexes in nephritis depends solely on the size of the complexes. Small complexes formed in antigen excess appear to localize on the epithelial side of the glomerular basement membrane, whereas larger complexes formed in antibody excess appear in a subendothelial mesangial position.

In human membranous nephropathy, immune complexes are found in the subepithelial space, whereas in MCGN the complexes appear in the mesangium and subendothelial space.

It may be that the type of renal lesion produced by tumour antigen-antibody complexes is related to the size of these complexes, which in turn depends to some extent on the pattern of antibody response of the patient to his tumour.

\section{References}

Costanza, M. E., Pinn, V., Schwartz, R. S., and Nathanson, L. (1974). Carcinoembryonic antigen-antibody complexes in a patient with colonic carcinoma and nephrotic syndrome. New Engl. J. Med., 289, 520-522. da Costa, C. R., Dupont, E., Hamers, R., Hooghe, R. 람 Dupuis, F., and Potvliege, R. (1974). Nephrotic syndrome in bronchogenic carcinoma: report of two cases with $\frac{\text { - }}{2}$ immunochemical studies. Clin. Nephrol., 2, 245-250.

Germuth, F. G., Jr. and Rodriguez, E. (1973). Immunopathology of the Renal Glomerulus. Little, Brown, Boston. Churchill/Livingstone, Edinburgh and London.

Hyman, L. R., Burkholder, P. M., Joo, P. A., and Segar, W. E. (1973). Malignant lymphoma and nephrotic $\vec{D}$ syndrome: a clinicopathologic analysis with light, immunofluorescence, and electron microscopy of the renal lesions. J. Pediat., 82, 207-217.

Lee, J. C., Yamauchi, H., and Hopper, J. Jr. (1966). The $\vec{\circ}$ association of cancer and the nephrotic syndrome. Ann. intern. Med., 64, 41-51.

Lewis, M. G., Loughridge, L. W., and Phillips, T. M. (1971). Immunological studies in nephrotic syndrome associated $\widehat{0}$ with extrarenal malignant disease. Lancet, 2, 134-135.

Loughridge, L. W. and Lewis, M. G. (1971). Nephrotic $\underset{\infty}{N}$ syndrome in malignant disease of non-renal origin. Lancet, 1, 256-259.

Muggia, F. M. and Ultmann, J. E. (1971). Glomerulonephritis or nephrotic syndrome in malignant lymphoma, $\rightarrow$ reticulum-cell type (Letter). Lancet, 1, 805. 